\title{
Efficient Operation of a Multi-functional Surface Mounting Device
}

\author{
Sungyeol Yu*, Jinhyeon Sohn*, Sungsoo Park* and Byung Jun Oh** \\ *Dept. of Industrial Engineering, Korea Advanced Institute of Science and Technology \\ 373-1 Gusong-dong, Yusong-gu, Taejon 305-701 Korea \\ ** Production Engineering Center, Samsung Electronics Co. \\ 416 Maetan3-dong, Paldal-gu, Suwon 442-742 Korea
}

\begin{abstract}
We consider the operation of a multi-functional surface mounting device(SMD) which mounts various components provided by diverse types of feeders such as cassette, stick, and tray on a printed circuit board(PCB). The problem is to minimize the time needed to assemble a PCB using the SMD. We decompose the problem into two subproblems, which are determining the allocation of component feeders and the mounting sequence of components, respectively. We propose heuristic algorithms to solve the subproblems and discuss the procedure to improve the solution. Computational results on real world problems are presented. (C) 1997 Elsevier Science Ltd
\end{abstract}

Kemwords : surface mounting device, feeder allocation, mounting sequence

\section{Introduction}

We consider the problem of minimizing the time needed to mount components on a printed circuit board(PCB) using a multi-functional surface mounting device(SMD). There have been many researches concerning the efficient operations of SMDs or automatic component insertion machines $[1,2,6,7,9]$. However, previous researches mostly have focused on the machines with specialized applications. The machines usually consist of head, table, and feeder which move independently, and used for mounting only special components, such as either small components or large components. Recently, SMDs with more versatilities have appeared which can handle almost all types of components such as large QFP components as well as small CHIP components. In these machines, the positions of the table and the feeders are fixed, and only the head moves to pick up and mount components. The operation characteristics of these new machines are different from the previous ones. In this paper, we present a heuristic algorithm to generate an efficient process plan for the machine.

The conceptual diagram of the SMD is given in Figure 1. The SMD is composed of a XY-robot having a head, four stages on which feeders are located, a vision camera to inspect components before mounting them, a nozzle storage and a control unit. The components are provided by diverse types of feeders such as cassette, stick, and tray feeders and each feeder supplies identical components. The cassette and stick feeders are located on lanes in stage 1,2 or stage 3 and the tray feeders are located in stage 4. The positions of PCB and feeders are fixed, and the head picks up a component from a feeder, moves to a mounting location on a PCB, and mounts it on the location. And, the head movement speed can be different compo- nent by component. Some components are required to be inspected by the vision camera before mounting them Feeders can be divided into some groups according to their types and whether components in the feeders use the vision camera or not. The number of lanes occupied by each feeder can be different. And, the nozzle used for each component can be different. The head has only one nozzle at a time, and it is changed, if necessary, during the operation.

The problem is to determine the allocation of component feeders on the lanes and the mounting sequence of the components concurrently so that the total mounting time is minimized. We decompose the problem into two subproblems, which find the allocation of component feeders and the mounting sequence of components.

The allocation problem of component feeders can be considered as the assignment problem with adjacency constraints which is NP-hard[8]. To solve the problem, we consider a measure which gives priorities to each feeder for allocation. The subproblem to find the mounting sequence of components when the feeder allocation is fixed can be formulated as a directed traveling salesman problem(TSP)[9]. For a solution approach of this subproblem we use the arbitrary insertion method. We apply the algorithms to real world problems

\section{A Mathematical Formulation}

The time needed to assemble a PCB is composed of the head movement time, picking-up time, mounting time and inspection time. Since the times needed for picking-up, mounting and inspection operations are constants and are independent of the locations of the feeders and the mounting sequence of the components, they are disregarded 


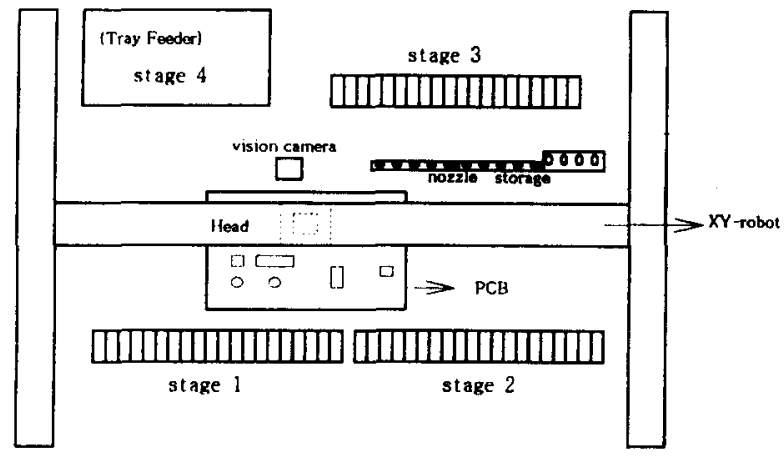

Figure 1. The conceptual diagram of the SMD

here. Now the total time needed to assemble a PCB can be regarded as the time taken by the head movement. The head movements can be further classified as the movements from a mounting location to a feeder, from a feeder to a mounting location, from a mounting location to the nozzle storage, from the nozzle storage to a feeder, from a feeder to the vision camera and from the vision camera to a mounting location. We assume that the time for the head movement from a final mounting location to the dummy location $O$ which indicates the point $(0,0)$, left upper corner on a PCB, is zero

Now, we present a mathematical formulation to minimize the total time needed for head movements. Let $P$ be the set of mounting locations including a dummy location $O$, $F$ be the set of feeders, and $L=\{1,2, \cdots, v\}$ be the set of lanes. We assume that $|l|$ is equal to or greater than the total number of feeders to be assigned. Binary decision variables $x_{i}$ and $y_{n}$ and arbitrary real variables $u_{i}$ are introduced. Then the problem can be formulated as follows

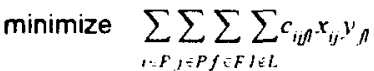

subject to

$$
\begin{aligned}
& \sum x_{i j}=1, \quad \forall j \in P \\
& \sum_{i \in P} x_{i j}=1, \quad \forall i \in P \\
& u_{i}-u_{j}+(n+1) x_{i j} \leq n, \quad \forall i_{1} j \in P \\
& x_{i j}=0 \text { or } 1, \quad \forall i, j \in P \\
& \sum y_{A}=1, \quad \forall f \in F \\
& \sum_{f \in f} v_{f} \leq 1, \quad \forall l \in l \\
& v_{f}+\sum_{f: F, f f l} v_{f, l} \leq 1 \quad \forall s_{f} \geq 2 \text { and } \forall l \in L,\{v\} \\
& v_{f}+\sum_{f^{\prime}: F^{\prime}(f)} y_{f^{\prime}, 1,1} \leq 1 \quad \forall s_{f} \geq 3 \text { and } \forall / \in L,\{1\} \\
& v_{A}+\sum_{f \in F \cup(f)} y_{f, l, 2} \leq 1 \quad \forall \cdot s_{f}=4 \text { and } \forall / \in l,\{v-1, v\} \\
& y_{A}=0 \text { or } 1, \quad \forall f \in F \text { and } \forall / \in l \text {. }
\end{aligned}
$$

where

\footnotetext{
$c_{i j h}=$ the time needed for head movement between two locations $i$ and $j$ when feeder $f$ supplies a component to mount on location $j$ and is assigned to the lane 1 . We assume that $c_{\text {iof }}=0$ for all locations $i \in P$, for all $f \in F$ and for all $l \in L$

$s_{f}=$ the number of lanes occupied by feeder $f$

$n=$ the number of mounting locations

$x_{i j}=1$ if and only if there exists a head movement from location $i$ to location $j$

$y_{A}=1$ if and only if feeder $f$ is assigned to lane $l$
}

The objective function (1) indicates the total time for head movements needed to assemble a PCB. Constraints (2)(5) are the traveling salesman problem tour restrictions. Constraints (2) and (3) force each node(mounting location) to be entered and to be left exactly once, and constraints (4) are subtour elimination constraints. Constraints (6), (7) and (11) are the constraints for a typical assignment problem. Constraints (6) imply that each component feeder should be assigned to exactly one lane, and constraints (7) imply that at most one component feeder can be assigned to each lane. If the number of physical lanes is equal to the number of lanes occupied by all feeders, we have equalities in (7). Constraints (8), (9) and (10) are called adjacent assignment constraints[8]. These are introduced when multiple lanes are occupied by a feeder.

In the above formulation, when the assignment variables $y_{f}$ are fixed, we have an asymmetric traveling salesman problem, the exact solution of which is computationally intractable. If the mounting sequence is fixed, the problem of determining the variables $y_{f}$ is an assignment problem with adjacent assignment constraints. The fact that the two subproblems are NP-hard $[3,8]$ shows that our problem belongs to the class of NP-hard problems. Since the number of components to be mounted and the number of feeders are large in our application, we consider heuristic algorithms to solve the subproblems.

\section{Heuristic Algorithm}

There are two types of movements in the head movement. One is the movement from a component feeder to a mounting location, and the other is from a mounting location to a component feeder. We will call the first as the required movement and the second as the nonrequired 
movement as they were referred in [2]. If the nozzles used for two components to be mounted consecutively are different, the time needed to move to the nozzle storage and to change the nozzles is included in the time for the nonrequired movement. If it is required to inspect a component by the fixed vision camera, the time needed to move the head to the vision camera is included in the required movement time. The problem to minimize the total time for head movements can be considered as the problem of minimizing the sum of the time needed for the required movement and the nonrequired movement for all mounting locations.

The algorithm consists of three phases. First we find the initial allocation of component feeders only considering the required movements. In the second phase, with the feeder locations being fixed, we determine the mounting sequence considering the nonrequired movements. Finally, we try to find improved solutions by revising the mounting sequence repeatedly with the pair-exchange of adjacent feeders. Detailed descriptions for each phase are given as follows

\section{1 initial allocation of component feeders}

The subproblem considered here is to determine the allocation of component feeders to minimize the time needed for all required movements. This can be regarded as the assignment problem which assigns the component feeders to available lanes. There exist not only typical assignment constraints but the constraints for adjacent lanes in our assignment problem, because the number of lanes occupied by each feeder can be different. For example, if the number of lanes occupied by a feeder is two, any other feeders cannot be assigned to an adjacent right lane of the lane which is occupied by that feeder. We propose a heuristic approach based on analysis of the time for the required movements.

Let $d$, be the distance from lane $/$ to the center point of a PCB. And let $I_{A}$ be the sum of the required movement time of the head from lane $l$ to the center of the PCB to mount all components supplied by feeder $f$, when feeder $f$ is assigned to lane $l$. The time $I_{A}$ is given by the following equation.

$$
i_{f}=n_{f} \frac{d_{i}}{\alpha_{f}}+n_{f} \cdot K=d_{f} \cdot w_{f}+n_{f} \cdot K
$$

where,

${ }_{s}=$ the number of components to be mounted on a board from feeder $f$

$\alpha_{f}=$ the head movement speed to mount a component in feeder $f$ on a board

$K=$ constant time to accelerate and decelerate the head

$w_{f}=\frac{n_{f}}{\alpha_{f}}($ weight of feeder $f)$

From the above equation (12), it can be easily shown that the following property holds

\section{<property>}

Suppose $d_{1}<d_{1}$, for two lanes $l_{1}$ and $l_{2}$ If $w_{f} \leq w_{g}$ for any distinct two feeders $f$ and $g$, then the following inequality holds.

$$
t_{A_{1}}+t_{s_{2}} \geq t_{A_{2}}+t_{s_{1}}
$$

proof : Let $\Delta=t_{A_{1}}-t_{f_{2}}$ and $\Delta g=t_{s_{1}}-t_{t_{2}}$ From equation (12), $\Delta f=\left(d_{1}-d_{i_{2}}\right) \cdot w_{f}$ and $\Delta g=\left(d_{i_{1}}-d_{i_{2}}\right) \cdot w_{g}$. Thus, $\Delta f-\Delta g=\left(d_{1}-d_{l_{2}}\right) \cdot\left(w_{f}-w_{g}\right) \geq 0$

By the above property, if we assume that all mounting locations exist at the center point on the $\mathrm{PCB}$, it is desirable that the feeder having more mounting locations and less movement speed is assigned to the nearest lane to the center of the board among available lanes. Actual mounting locations on a board are different each other and there exist the adjacency constraints in real world problems. Thus allocation of component feeders considering only the weight for each feeder does not guarantee the optimal solution to the problem to minimize the sum of the time for all the required movements. But we can obtain the solution approximately using the weight $w_{f}$ of feeder $f$. We, first, compute the weight of the feeders. And then, we select a feeder having the largest weight and assign that feeder to the nearest lane to the actual mounting locations of components in the feeder or to the vision camera position among current available lanes. We continue this procedure until all feeders are assigned to the lanes. We give the detailed description of the algorithm in the following

Algorithm 1: initial allocation of component feeders step 1) Compute the weight $w_{f}=n_{f} / \alpha$, for all $f \in F$

step 2) Select a feeder $f^{*}$, having the largest weight among feeders, not assigned yet

step 3) If components supplied by the feeder $f^{*}$ use the vision camera, assign $\dot{f}^{*}$ to the nearest lane to the vision camera among available lanes and go to step 5). Otherwise, go to step 4)

step 4) Find a lane $i^{*}=\min _{l}\left\{\sum_{p \in P_{i}} d(l, p), \quad \forall l \in L_{a}\right\}$

where $d(l, p)$ is the distance between lane $l$ and mounting location $p, L_{a}$ is currently available lane set, and $P_{1}$ is the set of locations to mount components supplied by the feeder assigned to lane $I$ Assign feeder $f^{\circ}$ to lane $i^{\circ}$.

step 5) If $F=\varnothing$, stop. Otherwise, $F=F \backslash\left\{f^{*}\right\}$ and go to step 2).

The feeders can be divided into some groups according to their characteristics. In this case, the grouping constraints exist, which imply that the same kind of feeders should be assigned to consecutive lanes. When the grouping constraints exist, we divide the feeder set $F$ into the subsets $F_{1}, F_{2} \cdots, F_{k}$ by their groups made by the grouping constraints. And, we define the group weight $w^{\prime}$, as the sum of the weights of feeders included in subset $F_{t}(t=1.2, \cdots, k)$, that is, $W_{t}=\sum_{f \in F_{t}} w_{f}$. We determine the relative lane space to assign the feeders included in the group for each group considering the group weight. And then, for each group we assign feeders in the group to 
predetermined lanes using algorithm 1

\subsection{Mounting sequence of components}

After the allocation of all component feeders are determined, the required movements are fixed. However, the nonrequired movements depend on mounting sequence because the route of the head is determined according to the next mounting location. Thus, given the allocation of component feeders, the subproblem to decide mounting sequence of components is to minimize the time for all nonrequired movements. A mounting sequence is defined as a sequence of locations on the board starting from the dummy point $O$ and ending at the dummy point $O$ This problem can be formulated as a directed TSP that finds the route to minimize the total nonrequired movements time, which passes all locations exactly once.

To solve the directed TSP in our application, we adopted the arbitrary insertion heuristic[5]. The cost coefficient between two nodes(locations) is given as the movement time needed to move a head from a location to the feeder which supplies a component to mount on the next mounting location, and, if necessary, it can include the time for movement to the nozzle storage. For the description of the algorithm, we define $d_{p q}$ be the distance from location $p$ to a feeder which supplies a component to mount on location $q$. We assume that $d_{p o}=0$ for all $p \in P$.

Now, the next is an algorithm to determine mounting sequence of components

\section{Algorithm 2 : mounting sequence of components}

step 1) Select a location $p$ among mounting locations of components supplied by the nearest feeder to the dummy location $O$, and construct a subtour $(O, p, O)$

step 2) If overall tour is constructed, stop. Otherwise, select an arbitrary location $r$ not included in current subtour.

step 3) For all edges (p.q) included in the current subtour, find an edge $\left(p^{\prime}, q^{\prime}\right)$ minimizing $d_{p r}+d_{r q}-d_{p q}$. By Inserting location $r$ between location $p^{\prime}$ and location $q^{\prime}$, update the subtour. Go to step 2)

\subsection{Comments on solution improvement}

The solutions for the above two subproblems do not guarantee the optimal solution to the overall problem. Thus, $A$ procedure to improve the initial solution is considered.

We used the pair exchange of feeders and the adjustment of mounting sequence to improve the initial solution as was given in [9]. When the exchange of feeder $f$ and feeder $g$, which are assigned to adjacent lanes and included in the same group, is considered, the locations for the feeders $f$ and $g$ are eliminated from the current mounting sequence and reinserted by the least time increment method[5] after exchanging two feeders. If the total time is reduced, we actually change the feeder $f$ and the feeder $g$. Then we get the new improved mounting sequence and proceed to the next pair exchange

\section{Test Results}

We applied the algorithm to a number of real industrial problems. In Table 1, we give data specifications of the problems. In addition, the table shows two actual result, one is the results performed by the existing control pro- gram and the other is the results performed by new control program loading the developed algorithms on the control unit in the SMD. Tests for our algorithm were performed on a Pentium $\mathrm{PC}(90 \mathrm{MHz})$ and the running time to get a final solution for each problem does not exceed $30 \mathrm{sec}$ onds. From the table, we can show that the time needed to mount a components is 1.60 seconds on average when use the new control program. On the contrary, the time needed to mount a component by the existing control program in the SMD is 1.84 seconds. The new control program reduce the mounting time per component about 13 percents compared to the existing program.

Table 1. Test results for real industrial problem (unit : seconds)

\begin{tabular}{|c|c|c|c|}
\hline $\begin{array}{c}\text { problem } \\
\text { number }\end{array}$ & $\begin{array}{c}\text { number of } \\
\text { feeders / } \\
\text { number of } \\
\text { locations }\end{array}$ & $\begin{array}{c}\text { time to assemble } \\
\text { a PCB } \\
\text { using the } \\
\text { exiting program }\end{array}$ & $\begin{array}{c}\text { time to assemble } \\
\text { a PCB } \\
\text { using } \\
\text { our algorithm }\end{array}$ \\
\hline 1 & $7 / 22$ & 76 & 73 \\
\hline 2 & $8 / 38$ & 107 & 68 \\
\hline 3 & $16 / 41$ & 100 & 79 \\
\hline 4 & $15 / 123$ & 207 & 178 \\
\hline 5 & $15 / 196$ & 282 & 274 \\
\hline
\end{tabular}

\section{Conclusions}

In this paper, we have considered the efficient operation of a multi-functional surface mounting device(SMD), which mounts various components on a printed circuit board As a solution approach, we have decomposed the problem into two subproblems; determining the allocation of feeder and determining the mounting sequence of components. And, we have proposed the heuristic algorithm for each subproblem, and discussed the procedure to improve the initial solution. Test results to the some real industrial problems show that our approach works well.

\section{References}

1. Ahmadi, J., Ahmadi, R., Matzo, H. and Tirupati, D., 1995, Component Fixture Positioning/Sequencing for Printed Circuit Board Assembly with Concurrent Operations, Operations Research, 37:444-457.

2. Ball, M. O and Magazine, M. J., 1988, Sequencing of Insertions In Printed Circuit Board Assembly, Operations Research, 36:192-201.

3. Bondy, J. A and Murty, U. S., 1985, Graph Theory with Applications, North-Holland, New York.

4. Laporte, G., 1992, The Traveling Problem: An Overview of Exact and Approximate Algorithms, European Journal of Operational Research, 59:231-247.

5. Lawler, E. L., Lenstra, J. K., Rinnooy Kan, A. H. K. and Shmoys, D.B., 1986, The Traveling Salesman Problem, John Wiley \& Sons, New York

6. Leipälä, T. and Nevalainen, O., 1989, Optimization of the Movements of a Component Placement Machine, European Journal of Operational Research, 38:167-177

7.Mettala, E. G and Egbelu, P. J., 1989, Alternative Approaches to Sequencing Robot Moves for PCB Assembly, International Journal of Computer Integrated Manufacturing, 2:243,256.

8. Mizuike, T. and Ito, Y., 1989, Optimization of Frequency Assignment, IEEE Transactions on Communications, 37:1031-1041

9. Sohn, J. and Park, S., 1996, Efficient Operation of a Surface Mounting Machine with a Multihead Turret, International Journal of Production Research, 34:1131-1143. 